\title{
Dietary cholesterol, plasma cholesterol and coronary heart disease
}

\author{
Michael Oliver ${ }^{1}$
}

Many who study cardiovascular epidemiology, nutrition, or cardiology have neither the will, the wish, nor the time to examine the fabric of the case for or against causal relationships. Opinions are set and deaf ears are turned. The polarization of views in the United States, for example, goes so far as to result in major court actions, alienation between doctors, disagreements within families, excessive advertising, and missionary zeal. Sometimes the advocates of a significant relation between, for example, dietary fat and coronary heart disease speak so loudly and so often that they endanger their own good case by appearing to be uncritical and to overlook inconvenient facts. Exactly the same is true of those who choose to disregard the links between dietary fat and coronary heart disease. I shall try to take the heat out of certain arguments by reappraising some of the facts, though anyone with the temerity to write on this subject is likely, at best, to be labelled injudicious. I shall focus on the relation between dietary cholesterol and coronary heart disease. But first dietary fat must be mentioned briefly.

\section{Dietary fat}

Some people equate the total amount of fat in the diet directly with mortality from coronary heart disease. Others consider that there is a definite, proved relation either between the saturated fat content or amount of cholesterol in the diet and a risk of developing coronary heart disease (Fig. 1). Several epidemiological studies indicate a positive correlation between the intake of saturated fat with the incidence of coronary heart disease. The most notable of these are the Seven Countries Study (Keys, 1970), the multiracial community survey in Cape Town (Bronte Stewart, Keys, and

1Address: Department of Cardiology, The Royal Infirmary, Edinburgh.

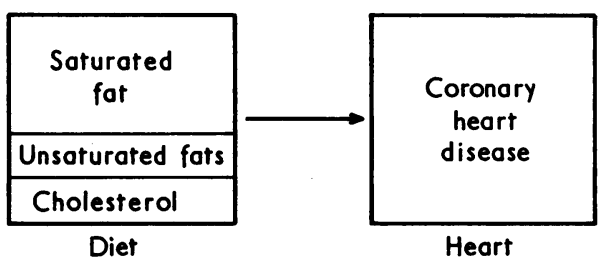

FIG. 1 A superficial view.

Brock, 1955), and studies of immigrants from poor to rich areas, where they were abruptly exposed to a higher standard of living than they had had previously (Toor et al., 1957; Larsen, 1957).

Most of the evidence has been derived from contrasts between comparatively primitive and rich communities, and there is no convincing evidence that there is a significant correlation between the intake of dietary saturated fat with mortality from coronary heart disease within so-called developed communities, where fat already comprises more than about 35 per cent of total calories. Moreover, it is not solely dietary saturated fat that is important: some have shown that the association is statistically stronger when animal protein is considered (Yerushalmy and Hilleboe, 1957) and when dietary sucrose is taken into account (Yudkin, 1957). These comments should not be interpreted as approbation for the high intake of saturated fat in the UK. There is little doubt that it is one of several important contributory influences on mortality, and as such the arguments set out in the recent COMA Report (1974) for a reduction of dietary saturated fat are surely correct.

\section{Dietary cholesterol}

The evidence incriminating dietary cholesterol as a cause of coronary heart disease in developed countries is virtually non-existent. Keys (1970) failed to show a significant relation from less well- 
developed to affluent countries for this dietary constituent.

Diets that are high in saturated fat are generally speaking, high in cholesterol. The ordinary mixed diet in the UK contains only between 10.5 and $15.5 \mathrm{mmol}$ (400 and $600 \mathrm{mg}$ ) cholesterol daily and the chief source is the egg, which contains $230 \mathrm{mg}$ cholesterol. Other common sources are milk, liver, shellfish, and meat. About 40 to 60 per cent of the cholesterol present in the food is absorbed, so that in very approximate terms $6 \mathrm{mmol}(250 \mathrm{mg})$ cholesterol enters the total body pool from dietary sources each day. Dietary cholesterol of this order has no measurable effect on plasma cholesterol. Extremes of cholesterol intake-excess (Connell, 1959) or none (Kempner, 1948) - do alter plasma levels but even these may have only a transient effect before a new homeostatic balance (see below) is achieved. With higher intakes the percentage of absorption falls and so a daily intake of about $78 \mathrm{mmol}(3 \mathrm{~g})$ can lead to the acquisition of no more than $26 \mathrm{mmol}(1 \mathrm{~g})$ per day. Probably the amount of cholesterol absorbed is affected by other constituents in the diet, particularly dietary fibre, by the form in which cholesterol is presented, and perhaps by genetic influences on absorption.

Dietary cholesterol is a contributant to plasma cholesterol, though not a major one, and that is about all that can be said about its relation to coronary heart disease.

\section{Plasma cholesterol}

The cholesterol circulating in the plasma represents about 10 per cent of the total body pool of cholesterol. There are only very weak correlations between total exchangeable body cholesterol and plasma cholesterol (Goodman and Noble, 1968). There are probably two large pools of tissue cholesterol with very low turnover rates which are independent of the rapidly exchangeable plasma pool (Samuel and Lieberman, 1973). The plasma level of cholesterol is determined by $a$ ) the amounts synthesized in the body, $b$ ) the rate of catabolism and excretion in the bile, $c$ ) the equilibration between plasma and tissues, and $d$ ) by the amount absorbed from the diet (Fig. 2). These are placed in order of probable importance.

Cholesterol synthesis and the enterohepatic circulation of bile acids form a vital homeostatic mechanism which influences critically the size of the cholesterol pool in the plasma as well as in the body. Bile acids are excreted into the duodenum, modified either by deconjugation or by other chemical changes through the action of bacteria, and some are reabsorbed unchanged in the ileum and returned in the blood to the liver. The rate of bile acid formation increases if the return to the liver is decreased and vice versa. This homeostatic nature of the enterohepatic circulation is very important in adjusting the body's reaction to variations in exogenous cholesterol intake and quite probably acts in a 'protective' role.

If these considerations are not enough to cause confusion in the interpretation of plasma cholesterol concentrations they are compounded by the fact that cholesterol is carried by a number of different lipoprotein fractions (World Health Organization, 1970). The availability and structure of various low density lipoproteins (Scanu, 1973)-and of the apoprotein associated with the binding of cholesterol-also determine the amount of cholesterol carried in the plasma, the duration of its transport, and its availability for catabolism or transfer to tissue; and the presence of high density lipoproteins may be important in relation to removal of cholesterol from tissue (Miller and Miller, 1975).

Genetic factors also influence plasma cholesterol concentrations. Plasma cholesterol levels show greater concordance in monozygotic twins compared with dizygotic twins (Pikkarainen, Takkunen,

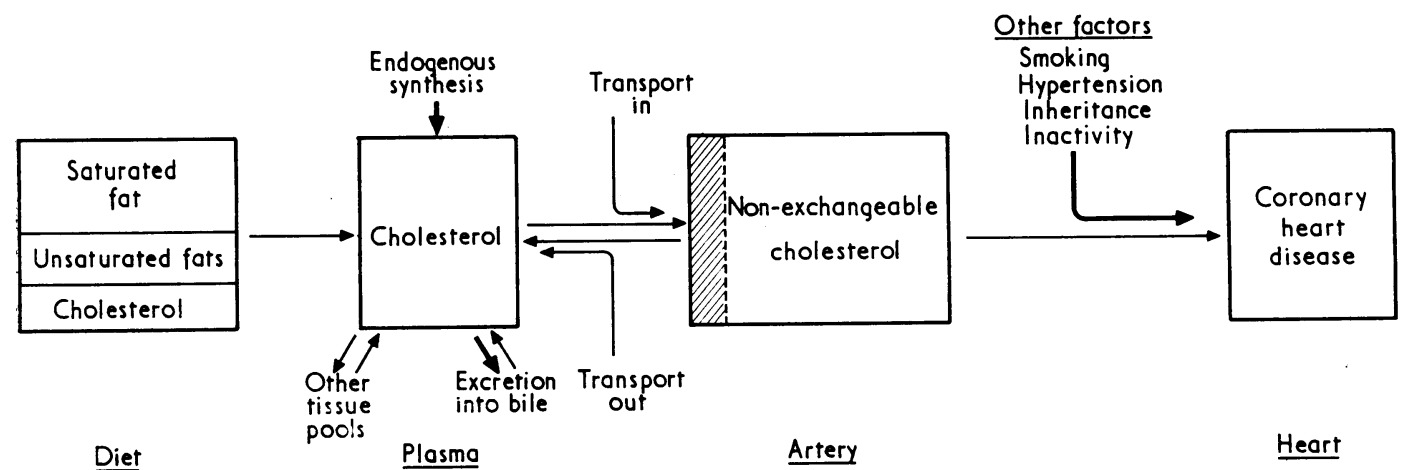

FI G. 2 Illustration of some other factors which should be taken into account when relating dietary cholesterol to coronary heart disease. 
and Kulonen, 1966). The inheritance of plasma cholesterol concentrations is greater from mother to child than from father to child and all first degree relationships show far greater similarity in plasma cholesterol than that between husband and wife (Schaefer, Adlersberg, and Steinberg, 1958).

The brief account-illustrated partly in Fig. 2of the controlling influences of plasma cholesterol should emphasize the extreme weakness of relating dietary cholesterol to any pathological process, let alone the clinical features such as coronary heart disease that are one stage removed from that process. The naiveté of placing much clinical and prognostic significance on particular levels of plasma cholesterol-unless very high indeed-in relation to the health of a given individual is also exposed.

\section{Arterial cholesterol}

Those who emphasize the importance of plasma cholesterol levels in man do so almost entirely on the positive epidemiological correlation with the incidence of coronary heart disease. The use of a derived end-point-namely, coronary heart disease or other ischaemic manifestations-and not atherosclerosis itself leads to confusion. It may be true that a moderate rise in plasma cholesterol levels in the region of 150 to $250 \mathrm{mg} / 100 \mathrm{ml}$, which occurs in most people in developed countries, is contributory to the almost universal presence of coronary atherosclerosis in middle-aged men in these countries, but there is no good evidence that such a rise by itself causes the clinical manifestations of angina, myocardial infarction, or sudden cardiac death.

The metabolism of the arterial wall (see Schettler and Weizel, 1974) is the critical intermediary. We do not know the quantitative relation between plasma and arterial cholesterol, and the process of cholesterol accumulation in the arterial wall is complex and not just dependent on concentration gradients. Low density lipoproteins pass directly into the aortic intima (Smith and Slater, 1973) and many factors influence their deposition (Walton, 1975). But the proportion of cholesterol-rich lowdensity lipoproteins in the plasma is only one side of the equation determining the extent of cholesterol deposition. Some will be catabolized and some will pass through to the veins and lymphatics of the adventitia. The arterial wall is probably protected from accumulation of cholesterol by active transport systems taking cholesterol out of arteries. For example, lecithin cholesterol acyltransferase (LCAT) is responsible for esterification of free cholesterol (Glomset and Norum, 1973) in the arterial wall. The activity of this enzyme is de- pendent on the availability of high density lipoproteins. Thus the plasma concentration of this lipoprotein may be critically important in determining the amount of cholesterol that can be removed from the arterial wall and ultimately excreted into bile acids.

It is surprising the extent to which other factors important in the genesis of coronary atherosclerosis are still ignored. The effects of increased intravascular pressure are well established, particularly experimentally, and in general the greater the increase the greater the deposition of cholesterol in the arterial wall (Smith and Slater, 1972). The role of fibrin-platelet thrombus formation is undoubtedly contributory to atherosclerosis, though still requiring clearer definition.

The most impressive evidence that pronounced hypercholesterolaemia is associated with an unusually high incidence of atherosclerotic vascular disease lies in the high incidence of this disease, and at a premature age, in individuals with the single gene-determined familial hypercholesterolaemia. The premature onset of coronary heart disease in those thought to be homozygous for this abnormality supports the view that disturbed cholesterol metabolism on a genetic basis plays a significant role. But there are plenty of reported cases of individuals heterozygous for familial hypercholesterolaemia surviving into middle or even late age, with a distinct rise in plasma cholesterol levels. Obviously there are other contributory factors important in determining the onset of coronary heart disease in these people but the contribution of familial hypercholesterolaemia to the total incidence of coronary heart disease is probably small. From the number of coronary deaths in the general population and the frequency with which heterozygotes for familial type IIA might occur, it has been calculated that the coronary death rate for men under 55 years attributable to the familial type II disorder is only 1:400-a surprisingly small contribution to the vast population problem (Slack, 1972).

Additional evidence often adduced to support the relation between high plasma cholesterol levels and atherosclerosis is that a rise in plasma cholesterol levels in animals may lead to arterial lesions, particularly aortic but also coronary in site. Many of these lesions are at best termed 'cholesterolosis' since they often lack, unless very gross, a major collagen response and also any element of plateletfibrin deposition. Regression studies in several species show that the removal of cholesterol previously added to an experimental diet is associated with a reduction in their lesions. Recent studies in monkeys are more impressive (Arm- 
strong, Warner, and Connor, 1970), though again highly artificial diets were used to induce lesions. That cholesterol-rich lesions can be induced by giving a diet abnormally high in cholesterol, and that these lesions can regress when this diet is removed, is a fact in itself and should be left as such. The contribution of dietary cholesterol to the normal mixed human diet is a fraction of what was given to these animals: the plasma cholesterol levels in man are a fraction of the levels produced in these animals: and the conditions do not mimic the exposure of man to slight rises of plasma cholesterol over many years. As yet the extrapolation of this information to man is unjustified.

It should be clear by now that we are getting further and further away from any direct relation between dietary cholesterol and coronary heart disease. Not only does the plasma have its own intrinsic controlling mechanisms which determine the amount of cholesterol present, but arterial metabolism itself determines the amount of cholesterol stored.

\section{Coronary heart disease}

The view that raised plasma cholesterol concentration is per se a cause of coronary heart disease is untenable. Cigarette smoking, hypertension, physical inactivity, inheritance, softness of drinking water, and other factors determine the step linking coronary atherosclerosis to coronary heart disease. The importance of these influences has been overshadowed by the cholesterol-coronary heart disease hypothesis, and it is interesting to reconsider the relative degrees of risk of some of these, as appraised in the Framingham data and presented in the Report of the Inter-Society Commission for Heart Disease Resources (1970). The increase in risk for mortality from coronary heart disease for Americans with a diastolic blood pressure higher than $105 \mathrm{mmHg}(14.0 \mathrm{kPa})$ is 2.2 times, for those smoking 20 cigarettes a day it is 1.9 times and for those with a serum cholesterol $>7.8 \mathrm{mmol}$ ( $>300$ $\mathrm{mg} / \mathrm{ml}$ ) it is 1.75 times. Those with the aggregated risk for excess cigarette smoking, raised diastolic blood pressure, and hypercholesterolaemia are 8.5 times more likely to have a major coronary event over a 10-year period compared with those not at risk but, even in these individuals, the incidence is only 1.7 per cent per year.

Looking at it another way, 83 per cent of such high-risk people are alive 10 years after identification. Perspective is helped when it is realized that about 1 in 12 of that population had serum cholesterol levels above $7.8 \mathrm{mmol} / 1$ (300 mg/100 $\mathrm{ml}$ ), and only 1 in 25 of these men died from coronary heart disease between the ages of 45 and
54 years. Applying these figures to the population in the United Kingdom it can be deduced that only 1 in 7 of the coronary deaths occurring at this age is associated with pronounced hypercholesterolaemia even though coronary deaths represent about 35 per cent of the total number of deaths.

\section{Conclusion}

Dietary cholesterol is not an important cause of coronary heart disease, and a moderate regular intake is not harmful.

But the high intake of saturated fat in developed countries is one of several factors contributing to coronary heart disease and, therefore, represents a health hazard. It is good sense, therefore, for healthy individuals to reduce the total amount of saturated fat and also the total calorie intake, which is often excessive.

\section{References}

Armstrong, M. L., Warner, E. D., and Connor, W. E. (1970). Regression of coronary atheromatosis in rhesus monkeys. Circulation Research, 27, 59.

Bronte-Stewart, B., Keys, A., and Brock, J. F. (1955). Serumcholesterol, diet, and coronary heart-disease. Lancet, 2, 1103.

COMA Report, Department of Health and Social Security (1974). Diet and Coronary Heart Disease. H.M.S.O., London.

Connell, W. F. (1959). Aetiology of atherosclerosis. British Medical fournal, 2, 358.

Glomset, J. A., and Norum, K. R. (1973). The metabolic role of lecithin: cholesterol acyltransferase: perspectives from pathology. Advances in Lipid Research, 11, 1.

Goodman, D. S., and Noble, R. P. (1968). Turnover of plasma cholesterol in man. Fournal of Clinical Investigation, 47, 231.

Kempner, W. (1948). Treatment of hypertensive vascular disease with a rice diet. American fournal of Medicine, $4,545$.

Keys, A. (1970). Coronary heart disease in seven countries. American Heart Association Monograph, No. 29.

Larsen, N. P. (1957). Diet and atherosclerosis. Archicies of Internal Medicine, 100, 436.

Miller, G. J., and Miller, N. E. (1975). Plasma-high-densitylipoprotein concentration and development of ischaemic heart-disease. Lancet, 1, 16.

Pikkarainen, J., Takkunen, J., and Kulonen, E. (1966). Serum cholesterol in Finnish twins. American fournal of Human Genetics, 18, 115.

Report of Inter-Society Commission for Heart Disease Resources (1970). Circulation, 42, A-55.

Samuel, P., and Lieberman, S. (1973). Improved estimation of body masses and turnover of cholesterol by computerized input-output analysis. Fournal of Lipid Research, 14, 189.

Scanu, A. M. (1973). The structure of human serum low- and high-density lipoproteins. In Atherogenesis: Initiating Factors. Ciba Foundation Symposium, No. 12, p. 223. Elsevier, Amsterdam.

Schaefer, L. E., Adlersberg, D., and Steinberg, A. G. (1958). Heredity, environment and serum cholesterol. Circulation, $17,537$.

Schettler, G., and Weizel, A. (1974). (Eds.) AtherosclerosisIII; Proceedings of the Third International Symposium. Springer, Berlin. 
Slack, J. (1972). The risk. In Lipid Abnormalities and Coronary Heart Disease Detection and Management. Report of a Symposium held at Royal Society of Medicine, 21 March 1972, pp. 8-12. Cavendish Medical Centre, London.

Smith, E. B., and Slater, S. S. (1972). Relationship between low-density lipoprotein in aortic intima and serum-lipid levels. Lancet, 1, 463.

Smith, E. B., and Slater, R. S. (1973). Lipids and low-density lipoproteins in intima in relation to its morphological characteristics. In Atherogenesis: Initiating Factors. Ciba Foundation Symposium, No. 12, p. 39. Elsevier, Amsterdam.

Toor, M., Katchalsky, A., Agmon, J., and Allalouf, D. (1957). Serum-lipids and atherosclerosis among Yemenite immigrants in Israel. Lancet, 1, 1270.
Walton, K. W. (1975). Factors affecting lipoprotein deposition in the arterial wall. In Blood and Arterial Wall in Atherogenesis and Arterial Thrombosis, p. 79. Ed. by J. G. A. J. Hautvast. E. J. Brill.

World Health Organization (1970). Classification of hyperlipidaemias and hyperlipoproteinaemias. Bulletin of the World Health Organization, 43, 891.

Yerushalmy, J., and Hilleboe, H. E. (1957). Fat in the diet and mortality from heart disease. New York State fournal of Medicine, 57, 2343.

Yudkin, J. (1957). Diet and coronary thrombosis: hypothesis and fact. Lancet, 2, 155.

Requests for reprints to Dr. M. F. Oliver, Department of Cardiology, The Royal Infirmary, Edinburgh EH3 9YW. 